\title{
Phoenix cells reduced database construction for efficient reflectarray synthesis
}

\author{
Andrea Guarriello \\ Heriot-Watt University \\ Edinbourgh, United Kingdom \\ a.guarriello@hw.ac.uk
}

\author{
Daniele Bresciani \\ Thales Alenia Space \\ Toulouse, France \\ daniele.bresciani@thalesaleniaspace.com
}

\author{
Renaud Loison \\ IETR \\ Rennes, France \\ renaud.loison@insa-rennes.fr
}

\author{
George Goussetis \\ Heriot-Watt University \\ Edinbourgh, United Kingdom \\ g.goussetis@hw.ac.uk
}

\author{
Herve Legay \\ Thales Alenia Space \\ Toulouse, France \\ herve.legay@thalesaleniaspace.com
}

\begin{abstract}
Reflectarray antennas are becoming a popular technology, in particular for what concerns space applications. In this paper a technique for the synthesis of low profile reflectarray for space application is proposed and demonstrated in the context of an S-band design. It consists of the construction of a reduced bijective database, obtained by selecting a very limited set of Phoenix cells with minimal frequency dispersion. The geometrical continuity requirement between cells presenting close phase shifts has to be ensured as well. This kind of database is particularly applicable to narrowband applications, which would include reflectors for scientific space missions.
\end{abstract}

Index Terms-Reflectarray, Phoenix Cells, reduced database.

\section{INTRODUCTION}

Reflectarray antennas are experiencing a growing interest in these last years thanks to their advanced features e.g. lowcost, low profile, conformal deployment, reconfigurability [1]. This makes reflectarrays a very attractive alternative to shaped reflectors for space applications. This is further evidenced from the latest NASA scientific missions ISARA [2] and $M a r C O$ [3], whose telecommunication subsystems are based on such technology. Space applications require more and more lightweight and low profile antennas, from where the need to design very thin reflectarray antennas having low dielectric losses.

In this paper, a strategy to build a bijective Phoenix cells [4] database that covers the full $360^{\circ}$ phase shift range in the presence of a very thin substrate is presented. The motivation of using higher order Phoenix cells (Fig. 1) stems from the requirement to reduce the substrate thickness (against the conventional $\lambda / 4$ used for Phoenix cells [4]) while maintaining low dielectric permittivity. Higher order cells indeed provide more flexibility in the RF response associated with the higher degrees of geometrical freedom. In order to avoid the very costly computation of a whole multidimensional database, the construction of a reduced database suitable for a reflectarray synthesis is proposed. The database is formally one-

This research is founded by the European commission through Marie Skłodowska-Curie actions H2020. dimensional, but it is composed of elements characterized by several degrees of freedom in the geometric patterns.

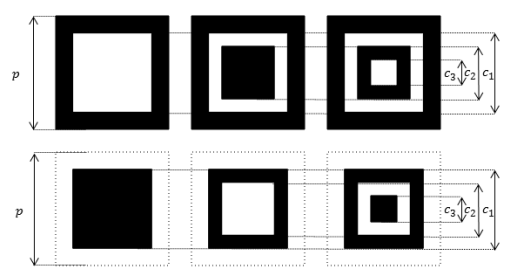

Fig. 1. From left to right Phoenix cells of first, second and third order, inductive type on the top, capacitive type on the bottom.

\section{REDUCED DATABASE CONSTRUCTION}

A. Complete database construction through Equivalent circuit model

A fast way to qualitatively characterize Phoenix cells is to consider an Equivalent Circuit (EC) model [5]. The advantage of this methodology is that the computation of the principal term of the reflection matrix is straightforward and very fast with respect to full-wave simulations unless the lack of high accuracy. By starting from the construction of first-order cells, with $c_{1} \in[0, p]$, being $\mathrm{p}$ the lattice size, the second order cells are built by considering a patch (or slot) inside the first slot (or patch) of size $c_{2} \in\left[0, c_{1}\right]$. The third order cells are by consequence built by considering a slot (or patch) inside the first patch (or slot) of size $c_{3} \in\left[0, c_{2}\right]$. The result is a database composed of roughly 100.000 cells. This computation is performed in a few minutes. The complete database is the departing point for the construction of a reduced database.

\section{B. Reduced database and optimization algorithm}

The reduced database is a subset of the complete database, which for each reflection phase provides the description of the unit cell with the most favourable performance. The phase shift supplied by each cell is computed by considering the cell as part of a periodic environment. Inevitably, a reflectarray 
cannot be a purely periodic structure, but a condition of quasiperiodicity is needed. In this sense, the smooth variation of the cells phase shift should be accompanied by a smooth variation of the geometry of the cells. The cells composing the database have to cover the phase shift range of $\left[-180^{\circ}, 180^{\circ}\right]$. In this particular case, the minimum dispersion cells are considered, in order to minimize the losses in the considered bandwidth. This feature can be particularly suitable for narrow-band applications.

Every cell is characterized by some parameters, as the geometric features that are described by a vector $\mathbf{c}=$ $\left[\begin{array}{lll}c_{1} & c_{2} & c_{3}\end{array}\right]$ (see Fig. 1). Another parameter that characterizes each cell is the phase dispersion in the band considered.

$$
\vartheta=\frac{\phi\left(f^{+}\right)-\phi\left(f^{-}\right)}{f^{+}-f^{-}}
$$

In equation $1, \phi\left(f^{ \pm}\right)$is the phase of the reflection coefficient at the upper and lower frequency band limits $f^{ \pm}$. The last parameter characterizing the cell is the typology, i.e. if the cell is of capacitive or inductive nature. The optimization algorithm can be therefore formalized:

$$
\begin{array}{rr}
\min & \alpha_{1_{i}}\left\|\mathbf{c}_{\mathbf{i}}-\mathbf{c}_{\mathbf{i}-\mathbf{1}}\right\|_{2}+\alpha_{2_{i}} \vartheta_{i} \\
\text { subject to } & \phi_{i}\left(f_{0}\right) \in\left[\phi_{0}+i, \phi_{0}+\Delta \phi+i\right]
\end{array}
$$

Where the subscript $i$ refers to the cell id, $i=1, \ldots, n-1 . \alpha_{1_{i}}$ and $\alpha_{2 i}$ are the weight parameters controlling the geometric continuity and the phase dispersion respectively, relative to the $i^{t h}$ cell, $\left\|\mathbf{c}_{\mathbf{i}}-\mathbf{c}_{\mathbf{i}-\mathbf{1}}\right\|_{2}$ is the Eucledian norm of the two consecutive cells geometries difference. $\phi_{0}$ is the first cell computed, for instance $\phi_{0}=-180^{\circ}$, i.e. a short circuit cell (full metal cell).

Once the reduced database is built from the cells characterized by an EC model, the selected geometries are stored and the complete scattering matrix of each cell is computed with commercial software (for instance HFSS or CST). The principal phase shifts obtained with a more accurate model are then sorted. In case the results are very different with respect to the results obtained with the EC model, a process of filtering is performed, in order to eliminate the cells that break the geometric continuity in the database, and interpolation is made in order to complete the missing phase shift range. Fig. 2 shows the reduced database final outfit computed with commercial software for a S-band database with central frequency $f_{0}=2.33 \mathrm{GHz}$ and $B W=1 \%$, with dielectric substrate $\epsilon_{r}=1.07-j 3.5 \cdot 10^{-3}$ and normal incidence. The lattice size is set to $p=\frac{\lambda}{3}$, while the substrate height is set to $h=\frac{\lambda}{13}$. The first cell on the bottom left supplies $\phi \approx-179^{\circ}$, the phase shift grows with $\Delta \phi=1^{\circ}$ from left to right and from the bottom to the top of the chart. The last cell is the one in the top right, with $\phi=180^{\circ}$. Fig. 3 shows the minimum dispersion profile vs the phase shift of the principal term of the reflection matrix of every cell composing the database.

\section{CONCLUSIONS}

The synthesis of a reduced Phoenix cell database allows a sensible reduction of the CPU time in the reflectarray synthesis process. Through a global optimization algorithm, it is possible to derive a set of limited cells able to cover the $360^{\circ}$ phase shift range with minimal dispersion and smooth geometric variations. The phase range coverage is guaranteed for all the typical incidence angle range we deal in a reflect-array design. These cells are the ingredient for the final synthesis of a layout, and it is particularly suitable for narrow-band applications.

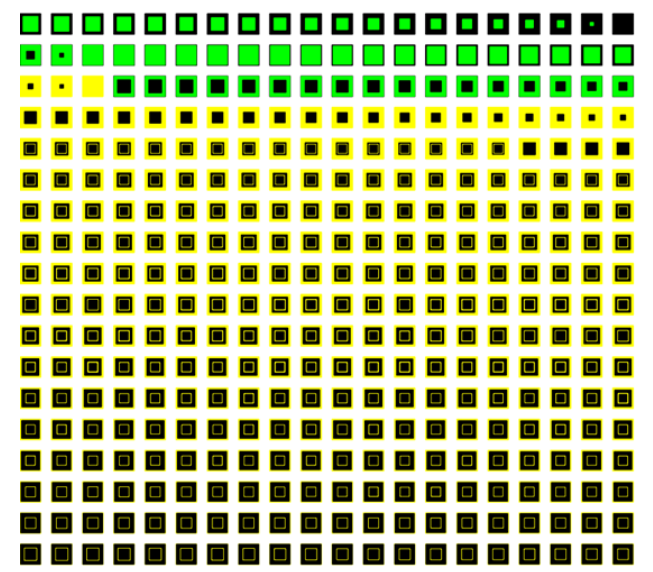

Fig. 2. Phoenix cells reduced database. In yellow the capacitive cells, in green the inductive cells.

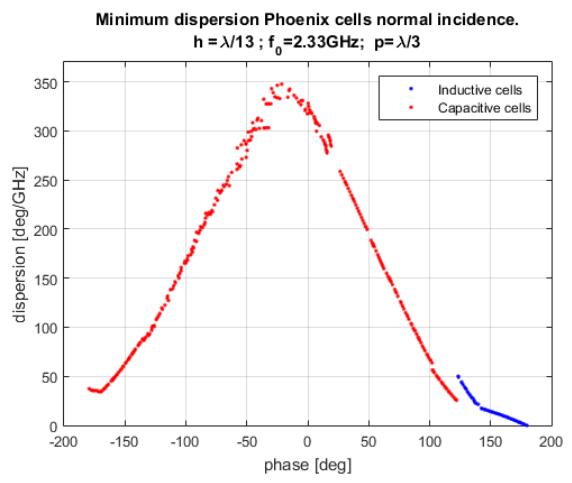

Fig. 3. Frequency dispersion $v s$ phase-shift of the complete reduced database.

\section{REFERENCES}

[1] J. Huang,and J. A. Encinar, Reflectarray antennas, vol. 30). John Wiley \& Sons, 2007, pp. 1-26.

[2] R. E. Hodges, M. J. Radway, A. Toorian, D. J. Hoppe, B. Shah, and A. E. Kalman, "ISARA-integrated solar array and reflectarray CubeSat deployable Ka-band antenna." 2015 IEEE International Symposium on Antennas and Propagation \& USNC/URSI National Radio Science Meeting, pp. 2141-2142, 2015.

[3] R. E. Hodges, N. Chahat, D. J. Hoppe, and J. D. Vacchione, "A Deployable High-Gain Antenna Bound for Mars: Developing a new folded-panel reflectarray for the first CubeSat mission to Mars." IEEE Antennas and Propagation Magazine, vol. 59(2), pp. 39-49, 2017.

[4] L. Moustafa, R. Gillard, F. Peris, R. Loison, H. Legay, and E. Girard, "The phoenix cell: A new reflectarray cell with large bandwidth and rebirth capabilities." IEEE Antennas and wireless propagation letters, vol 10, pp. 71-74, 2011.

[5] O. Luukkonen, C. Simovski, G. Granet, G. Goussetis, D. Lioubtchenko, A. V. Raisanen, and S. A. Tretyakov, "Simple and accurate analytical model of planar grids and high-impedance surfaces comprising metal strips or patches." IEEE Transactions on Antennas and Propagation, vol. 56(6), pp. 1624-1632, 2008 